\title{
Pseudo-Differential Filter Design Using Novel Adjustable Floating Inductance Simulator with Electronically Controllable Current Conveyors
}

\author{
Roman Sotner ${ }^{1,2}$, Norbert Herencsar ${ }^{2}$, Jan Jerabek ${ }^{2}$, Aslihan Kartci ${ }^{1,2}$, Jaroslav Koton ${ }^{2}$, Tomas Dostal ${ }^{3}$ \\ ${ }^{1}$ Department of Radio Electronics, Faculty of Electrical Engineering and Communication, \\ Brno University of Technology, \\ Technicka 12, Brno, 616 00, Czech Republic \\ ${ }^{2}$ Department of Telecommunications, Faculty of Electrical Engineering and Communication, \\ Brno University of Technology, \\ Technicka 12, Brno, 616 00, Czech Republic \\ ${ }^{3}$ Department of Technical Studies, College of Polytechnics Jihlava, \\ Tolsteho 16, Jihlava 586 01, Czech Republic \\ sotner@feec.vutbr.cz
}

\begin{abstract}
This paper presents new floating inductance simulator based on three electronically controllable current conveyors (ECCIIs) and differential voltage buffer (DVB). The inductance simulator offers simple electronic control of inductance value as well as simple control of losses by change of parameters of active elements. Based on this floating inductance simulator, example of reconnection-less reconfigurable pseudodifferential first-order filter, allowing change of transfer function between high-pass and inverting all-pass response, is discussed and studied. Moreover, the proposed structure can be easily extended to a pseudo-differential second-order band-bass filter. Behaviour of presented solutions is verified by PSpice simulations and also experimentally in frequency band up to $10 \mathrm{MHz}$. Obtained results confirmed expected features.
\end{abstract}

Index Terms-Differential active filter; differential mode; pseudo-differential-mode; electronic control; inductance simulator; reconfigurability.

\section{INTRODUCTION}

In general, fully-differential (symmetrical) circuits have many applications due to specific features they offer, i.e. attenuation of common-mode noise as well as better rejection of signals incoming from power supply sources and possibly also better dynamics in comparison to single-ended circuitries [1]-[4]. However, increased complexity of circuitry is cost for these benefits. Electronic controllability of parameters of analogue and mixed-mode circuits is useful in single-ended as well as in case of differential-mode operation. Therefore, circuit solutions allowing these features are subjects of interest of many researchers [4]. Digital control of important parameters of the differential-

Manuscript received 23 December, 2016; accepted 18 March, 2017.

Research described in this paper was financed by Czech Ministry of Education in frame of National Sustainability Program under grant LO1401. For research, infrastructure of the SIX Center was used. Research described in the paper was supported by Czech Science Foundation projects under No. 16-11460Y. filter was also investigated in the past [5].

Division of differential systems (for example see [1]-[8]) to truly-differential and so-called pseudo-differential-mode of operation, is perfectly explained in [8] which brings new insight into this research topic. Note that complexity of pseudo-differential-mode circuits is significantly reduced in comparison to fully-differential-mode solutions due to partially grounded subparts of differential systems, i.e. input and output ports are differential whereas their inner circuitry consists of grounded (analog ground) passive components and terminals. Many from previously reported pseudodifferential circuit solutions were focused on biquadratic (second-order) active filters, [6]-[9] for instance. Electronic controllability [6] as well as multi-functionality (providing several transfer responses) [7] were also studied in the past.

In this paper we are introducing a novel solution of floating inductance simulator allowing features not reported in previous works. These features are beneficial especially in electronic reconfiguration of transfer function and electronic control of pseudo-differential-mode filter parameters. Here proposed filtering applications can be divided to two groups: (i) first-order reconfigurable filter, (ii) second-order bandpass filter. Comparison of selected solutions of first-order active filters in single-ended, fully-differential, or pseudodifferential form [10]-[14] is available in Table I. In general, many of these have multifunctional features, however, features such as reconnection-less reconfigurability (possible to obtain various transfer responses without change of input/output terminal) were studied only in single-ended filtering solutions (see for example [15]). To the best of authors' knowledge, circuits allowing reconfiguration of transfer responses, i.e. certain multi-functionality of the filter between at least two different types of fully-differential or pseudo-differential filters were not reported in the open literature yet. Here presented structure of floating inductance simulator allows such electronic reconfiguration, if its 
implementation is appropriate, as it is shown in this paper.

TABLE I. COMPARISON OF REPORTED PSEUDO/FULLYDIFFERENTIAL FIRST-ORDER MULTIFUNCTIONAL FILTERS

\begin{tabular}{|c|c|c|c|}
\hline Work & $\begin{array}{c}\text { No. of pas./act. } \\
\text { elements }\end{array}$ & $\begin{array}{c}\text { Available } \\
\text { functions }\end{array}$ & $\begin{array}{c}\text { Transfer response } \\
\text { configurability }\end{array}$ \\
\hline$[10]$ & $4-6 / 1$ & AP, BR & No \\
\hline$[11]$ & $2-6 / 1-2$ & LP, HP, AP & No \\
\hline$[12]$ & $4 / 1$ & AP & No \\
\hline$[13]$ & $4 / 2$ & AP, LP & No \\
\hline$[14]$ & $1 / 3$ & AP & No \\
\hline Fig. 3 & $2(6) / 1(4)$ & HP, iAP & Yes \\
\hline
\end{tabular}

Note: (i) DT - (inverting) direct transfer; (i) $A P$ - (inverting) all-pass; $L P$ - low-pass; (i) HP - (inverting) high-pass; BR - band reject.

\section{DESCRIPTION OF FLOATING INDUCTANCE SiMULATOR}

Proposed floating inductance simulator shown in Fig. 1 employs three electronically adjustable current conveyors of second generation (ECCIIs) [16] described by the following inter-terminal transfers: $I_{\mathrm{Z}}=B . I_{\mathrm{X}}, V_{\mathrm{X}}=V_{\mathrm{Y}}, I_{\mathrm{Y}}=0$ and one differential voltage buffer (DVB) [16] providing the following operation: $V_{+}-V_{-}=V_{\text {out }}$. Note that the current gain $B$ of each ECCII is controllable linearly by DC voltage $\left(B \cong V_{\text {SET_B }}\right)$. Commercially available devices EL2082 and AD830 are examples of previously discussed elements (ECCII, DVB) with well-fitting behaviour up to several tens of $\mathrm{MHz}$. Assuming $R_{\mathrm{x} 1}=R_{\mathrm{x} 2}=R_{3}$ are equal and designated as $R_{\mathrm{L}_{-} \text {int }}$, the admittance description (based on method of unknown nodal voltages: $\boldsymbol{I}=\boldsymbol{Y} \times \boldsymbol{V}$ ) of the circuit in Fig. 1 presents set of equations:

$$
\left[\begin{array}{c}
I_{\text {inp } 1} \\
I_{\text {Inp } 2}
\end{array}\right]=\left[\begin{array}{cc}
a^{-1} B_{1} & -a^{-1} B_{1} \\
-a^{-1} B_{2} & a^{-1} B_{2}
\end{array}\right]\left[\begin{array}{c}
V_{\text {inp } 1} \\
V_{\text {inp } 2}
\end{array}\right],
$$

where $a=s C_{L_{-} \text {int }} R_{L_{-} \text {int }}^{2}+\left(2-B_{3}\right) R_{L_{-} \text {int }}$.

Then, assuming $B_{1}=B_{2}=B_{1,2}$, impedance of equivalent inductance emulator has the following theoretical form

$$
\begin{gathered}
L_{e q}(s)=s L_{e q \_ \text {int }}+R_{s_{-} \text {int }}= \\
=\frac{s C_{L_{-} \text {int }} R_{L_{-} \text {int }}^{2}}{B_{1,2}}+\frac{\left(2-B_{3}\right) R_{L_{-} \text {int }}}{B_{1,2}},
\end{gathered}
$$

where $C_{\mathrm{L}_{\mathrm{i}} \text { int }}$ and $R_{\mathrm{L}_{-} \text {int }}$ are passive elements of the inductance simulator and it is clear that the proposed circuit behaves as lossy inductance. However, the lossy part $R_{\mathrm{S}_{\mathrm{S}} \text { int }}$ in (1) can be easily eliminated by selecting current gain $B_{3}\left(\mathrm{ECCII}_{3}\right)$ equal to 2 , losses are negative for $B_{3}>2$. Nevertheless, lossy part of the proposed inductance simulator can be also beneficial for application as introduced later. Moreover, it is obvious that value of $L_{\mathrm{eq}}$ int can be controlled electronically by $B_{1}$ and $B_{2}$.

Proposed inductance simulator in Fig. 1 was designed for further implementations with the following values of internal elements: $\quad R_{\mathrm{L}_{-} \text {int }}=560 \Omega, C_{\mathrm{L}_{-} \text {int }}=470 \mathrm{pF}$ as a particular example. The PSpice simulation results of the inductance simulator in grounded form are shown in Fig. 2. Inductance value $L_{\text {eq }}$ was changed from $2.63 \mu \mathrm{H}$ to $0.13 \mu \mathrm{H}$ by $V_{\text {SET_B } 1,2}$ between 0.1 and $2 \mathrm{~V}$, i.e. gains $B_{1}$ and $B_{2}$ are between 0.1 and 2 .

\section{IndUCTANCE SimULATOR IMPLEMENTATION IN PSEUDO- DIFFERENTIAL FILTERING APPLICATIONS}

Benefits of the proposed solution were verified in several filtering applications shown in the following sections. Inductance simulator is used as floating element in RL or RLC ladder structures. Theoretical expectations are supported by simulation results and also by laboratory experiments.

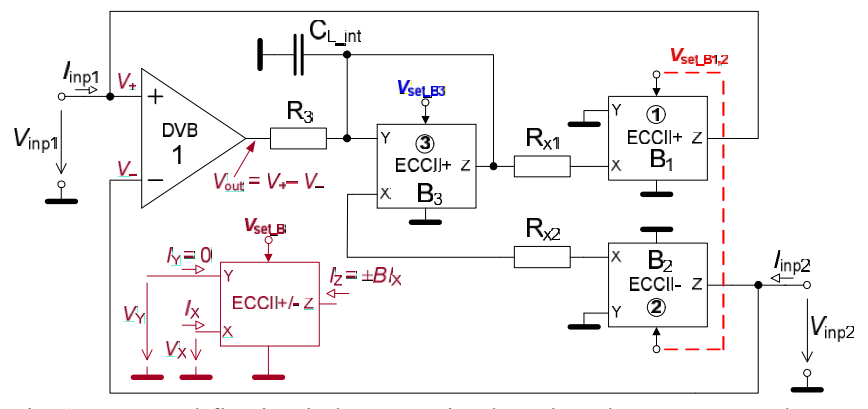

Fig. 1. Proposed floating inductance simulator based on ECCIIs and DVB including description of behaviour of active elements (brown colour).

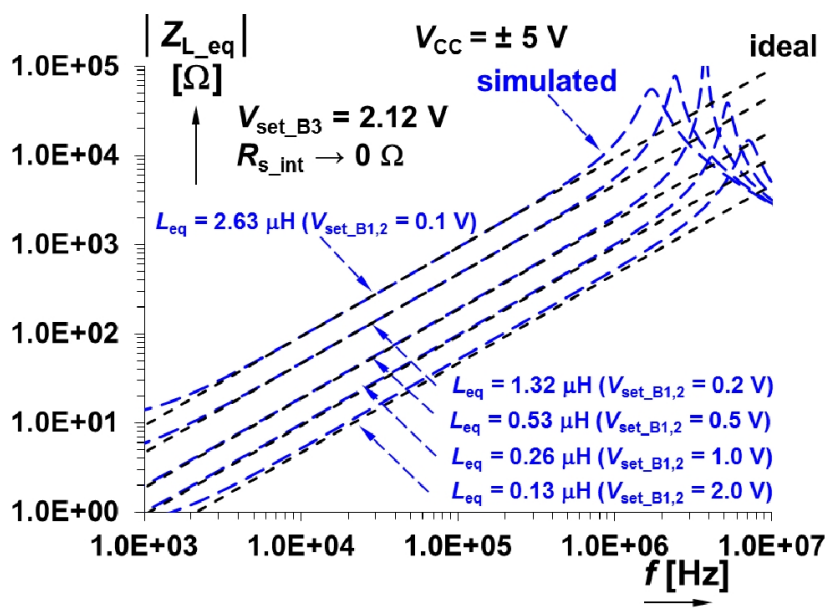

Fig. 2. Simulation results of impedance characteristics (magnitude) of proposed inductance simulator (Fig. 1) in lossless mode while tuning $B_{1,2}$.

\section{A. Reconfigurable First-Order High-Pass/Inverting All- Pass Filter}

Proposed circuit in Fig. 3 has a pseudo-differential configuration, which, in accordance to [8], means that it has differential input/output terminals, but inner circuit itself is not fully symmetrical. Special features of the introduced inductance simulator in Fig. 1 are very useful for reconnection-less reconfigurability of transfer function in pseudo-differential first-order high-pass/inverting all-pass (HP/iAP) filter. These features are not available if any standard solution of simulator of lossless inductance is employed as $L_{\mathrm{eq}}$ in structure shown in Fig. 3.

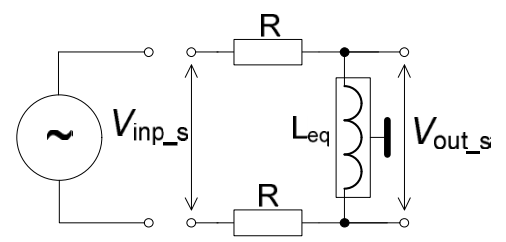

Fig. 3. Implementation of the floating inductance simulator in pseudodifferential-mode reconfigurable first-order $\mathrm{HP} / \mathrm{iAP}$ filter $\left(V_{\text {inp/out_s }}-\right.$ symmetrical input/output voltage).

Differential-mode transfer function (full description (1) 
applied) of the circuit in Fig. 3 has symbolical form

$$
\begin{gathered}
K_{H_{P_{-} i A P}}(s)=\frac{V_{\text {out_s }} s}{V_{\text {inp_s }} s}= \\
=\frac{2\left(s+\frac{2-B_{3}}{C_{L_{-} \text {int }} R_{L_{-} \text {int }}}\right)}{s+\frac{R_{L_{-} \text {int }}\left(2-B_{3}\right)+R\left(B_{1}+B_{2}\right)}{C_{L_{-} \text {int }} R_{L_{-} \text {int }}^{2}} .}
\end{gathered}
$$

Transfer function (3) offers high-pass (HP) response, if $B_{3}=2$

$$
K_{H P}(s)=2 \frac{s}{s+\frac{R\left(B_{1}+B_{2}\right)}{C_{L_{-} \text {int }} R_{L_{-} \text {int }}^{2}}},
$$

where cut-off (pole) frequency is defined by

$$
\omega_{p 1}=\frac{R\left(B_{1}+B_{2}\right)}{R_{L_{-} \text {int }}^{2} C_{L_{-} \text {int }}} .
$$

The easiest example of inverting all-pass (iAP) response is available for $R=R_{\mathrm{L}_{-} \text {int }}, B_{1}=B_{2}=B_{1,2}=1, B_{3}=3$, from (3) we obtained simplified form of transfer

$$
K_{i A P}(s)=2 \frac{s-1 /\left(C_{L_{-} \text {int }} R\right)}{s+1 /\left(C_{L_{-} \text {int }} R\right)} .
$$

The following relation must be valid for $B_{3}$ if iAP response is requested

$$
B_{3}=2+\frac{B_{1}+B_{2}}{2} \text {. }
$$

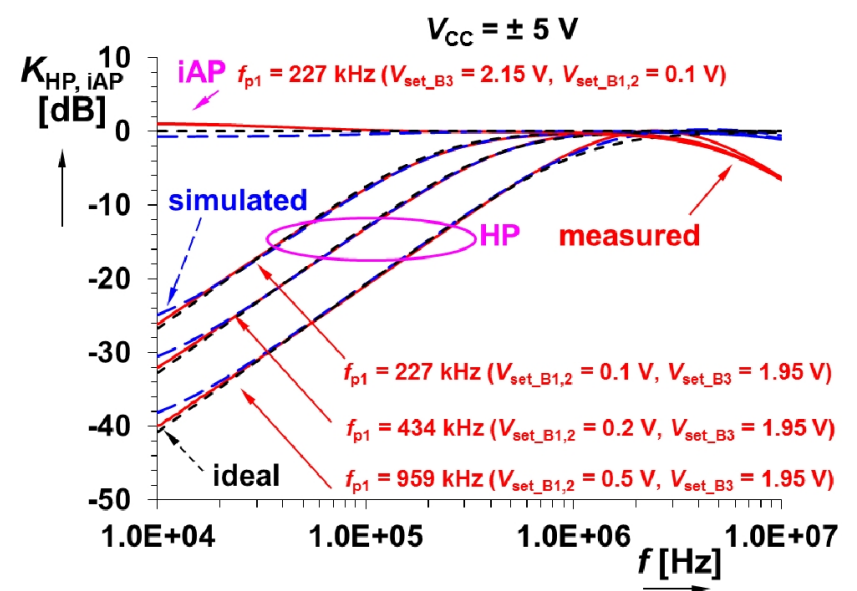

Fig. 4. Magnitude responses of the pseudo-differential-mode reconfigurable HP/iAP filter reconfigured by control of $V_{\text {set_B3 }}$ and several examples of tuning of the cut-off frequency of HP response by $V_{\text {set_B1,2. }}$.

Both pole/zero frequency has simple form $\omega_{\mathrm{p} 1}=1 / R C_{\mathrm{L}_{-} \text {int }}$. Also note that pole frequency can be controlled electronically by $V_{\text {set_B } 1,2}$ and type of transfer function is reconfigured by loss part of inductor simulator ( $B_{3}$ gain). Figure 4 includes comparison of experimental and simulated results of the filter from Fig. 3, when working resistor was set $R=1 \mathrm{k} \Omega$. Resulting traces were obtained for ideal cut-off frequency equal to $216 \mathrm{kHz}$ (simulated $226 \mathrm{kHz}$, measured $227 \mathrm{kHz})$ obtained by $V_{\text {set_B1,2 }}=0.1 \mathrm{~V}, V_{\text {set_B3 }}=1.95 \mathrm{~V}$ (HP response) or $2.15 \mathrm{~V}$ in case of iAP response ((7) for $\left.B_{3}\right)$. The example of $\omega_{\mathrm{p} 1}$ tuning in case of the HP response by control of $B_{1,2}$ can be observed also in Fig. 4. In measurements the change of $V_{\text {set_B1,2 }}$ between 0.1 and $0.5 \mathrm{~V}$ ensures adjustment of $f_{\mathrm{p} 1}$ from $227 \mathrm{kHz}$ to $959 \mathrm{kHz}$, respectively.

\section{B. Pseudo-Differential-Mode Second-Order Band-Pass Filter}

This application is easily available by adding a capacitance between output terminals as shown in Fig. 5. Transfer function of ideal RLC ladder structure employing lossless $L_{\text {eq }}$ considered in fully-differential form (1) is defined as

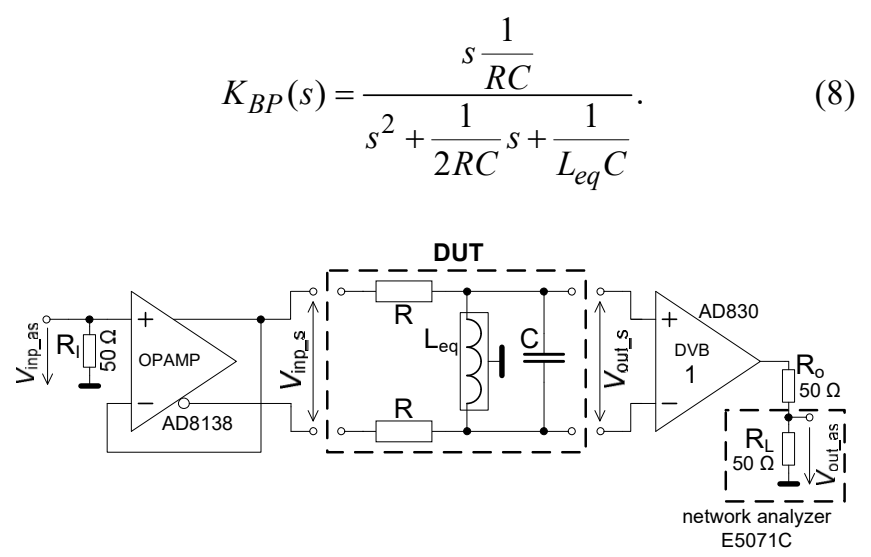

Fig. 5. Implementation of the inductance simulator in second-order RLC ladder differential band-pass filter. Schematic includes experimental setup ( $V_{\text {inp/out as }}$ - asymmetrical voltage; $V_{\text {inp/out_s }}-$ symmetrical voltage).

Considering the implementation of lossless $L_{\text {eq_int }}$ (i.e. $B_{3}=2$ ), transfer function (8) changes to

$$
K_{B P}(s)=\frac{s \frac{1}{R C}}{s^{2}+s \frac{1}{2 R C}+\frac{B_{1}+B_{2}}{2 R_{L_{-} \text {int }}^{2} C_{L_{-} \text {int }} C}},
$$

where center (pole) frequency and quality factor have the following expressions:

$$
\begin{gathered}
\omega_{p 2}=\frac{1}{R_{L_{-} \text {int }}} \sqrt{\left.\frac{B_{1}+B_{2}}{2 C_{L_{-} \text {int }} C} \Rightarrow \omega_{p 2}\right|_{B_{1}=B_{2}=B_{1,2}}=} \\
=\frac{1}{R_{L_{-} \text {int }}} \sqrt{\frac{B_{1,2}}{C_{L_{-} \text {int }} C}}, \\
Q=\frac{2 R}{R_{L_{-} \text {int }}} \sqrt{\left.\frac{\left(B_{1}+B_{2}\right) C}{2 C_{L_{-} \text {int }}} \Rightarrow Q\right|_{B_{1}=B_{2}=B_{1,2}}=} \\
=\frac{2 R}{R_{L_{-} \text {int }}} \sqrt{\frac{B_{1,2} C}{C_{L_{-} \text {int }}}} .
\end{gathered}
$$

Note that this second-order BP filter allows tuning of the pole frequency with theoretically no effect on bandwidth, because $B W[\mathrm{~Hz}]=1 /(4 \pi R C)$, and also change of quality 
factor without change of gain during the tuning process. Note that both filters (Fig. 3 and Fig. 5) have +6 dB passband gain (see const. 2 in (3) and (9)). However, experimental setup (output divider $R_{\mathrm{O}}$ and $R_{\mathrm{L}}$ of network analyser) performs division by 2 , therefore, ideal pass-band gain of the whole system is $0 \mathrm{~dB}$.

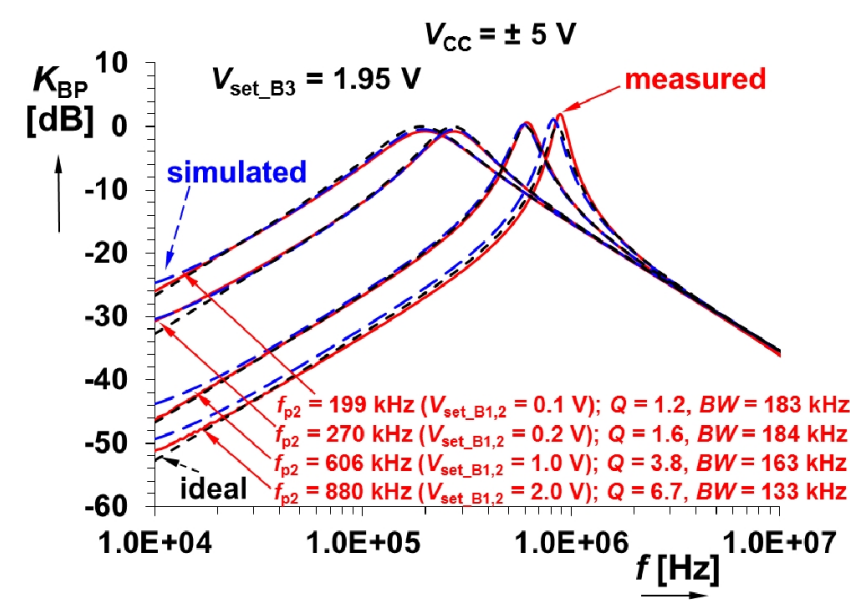

Fig. 6. Magnitude responses of the differential-mode second-order BP filter during tuning of the center frequency.

Comparison of experimental results, simulations, and theoretical traces of magnitude and phase responses for the pseudo-differential-mode BP filter is given in Fig. 6. Passive component values and active parameters were set as follows: $R_{\mathrm{L}_{-} \text {int }}=560 \Omega, C_{\mathrm{L}_{-} \text {int }}=C=470 \mathrm{pF}, R=1 \mathrm{k} \Omega, B_{3}=0$ and $B_{1}=B_{2}=B_{1,2}=1 \quad\left(V_{\text {set_B } 1,2}=1 \mathrm{~V}\right) . \quad$ Theoretical, experimentally tested, and simulated BP achieved $f_{\mathrm{p} 2}=\{605$, 606 , and 596$\} \mathrm{kHz}$, with $B W=\{169,163$, and 163$\} \mathrm{kHz}$ and $Q=\{3.6,3.8$, and 3.7$\}$ respectively. Tuning process (adjustment of $B_{1,2}$ ) can be also observed in Fig. 6. In measurements, adjusting of $V_{\text {set_B1,2 }}$ between $0.1 \mathrm{~V}$ and $2 \mathrm{~V}$ offers range of $f_{\mathrm{p} 2}$ tuning from $199 \mathrm{kHz}$ to $880 \mathrm{kHz}$. All details are noted in figures. Presented experimental results in Fig. 4 and Fig. 6 were carried out using vector network analyser E5071C with test setup given in Fig. 5. Figure 7 shows the fabricated prototype including converters from single-ended to differential mode and vice versa serving for performed experimental tests.

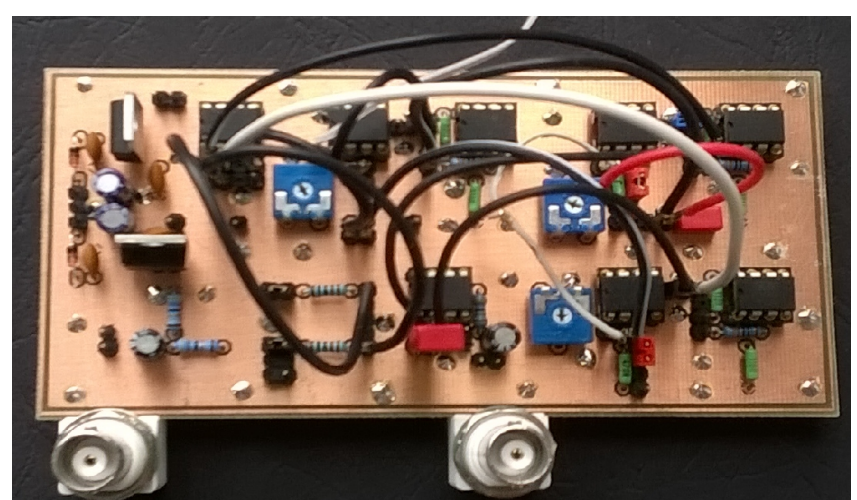

Fig. 7. Fabricated experimental prototype of pseudo-differential filter.

\section{DisCUSSION AND SUMMARIZATION OF RESUlTS}

Analysis of discussed inductance simulator revealed following advantages: a) possible implementation in differential applications (floating element), b) pseudo- differential character of circuitry (inner grounded capacitor), c) simple electronic controllability of inductance value and losses including special form of losses (2) that allows interesting applications. Note that these features are not simultaneously available in many standard solutions. The value of designed inductance simulator was tested from $2.63 \mu \mathrm{H}$ to $0.13 \mu \mathrm{H}$ ( $V_{\text {set_B1,2 }}$ adjusted from $0.1 \mathrm{~V}$ up to 2.0 $\mathrm{V})$, i.e. tunability ratio is approximately 1:20.

In accordance to the state-of-the-art discussed in Table I, the first application example of the designed special inductance implementation (reconfigurable first-order filter) represents unique device, because up to now (pseudo)differential-mode reconfigurable filters have not been widely investigated. Operation of the HP/iAP was verified by change of $V_{\text {set_B3 }}$ from $1.95 \mathrm{~V}$ to $2.15 \mathrm{~V}$ $(\mathrm{HP} \rightarrow \mathrm{iAP})$. Tuning range of the pole frequency yields values from $227 \mathrm{kHz}$ to $959 \mathrm{kHz}$ ( $V_{\text {set_B1,2 }}$ set from $0.1 \mathrm{~V}$ to $0.5 \mathrm{~V})$. The second example of application, the pseudodifferential second-order BP was tested in frequency range from $199 \mathrm{kHz}$ to $880 \mathrm{kHz}$ ( $V_{\text {set_B1,2 }}$ set from $0.1 \mathrm{~V}$ to $2.0 \mathrm{~V}$ ) with almost constant bandwidth approximately equal to $165 \mathrm{kHz}$.

\section{CONCLUSIONS}

Presented floating inductance simulator was tested in two novel applications namely pseudo-differential-mode reconfigurable first-order $\mathrm{HP} / \mathrm{iAP}$ filter and second-order BP filter derived from passive prototypes. Due to limitations of commercially available devices used in inductance simulator, targeted frequency range was in hundreds of $\mathrm{kHz}$. Experimental results are in good agreement with simulations and theory. Proposed inductance simulator offers interesting features in given applications and special form of its losses (in lossy mode of operation) brings useful implementations also in pseudo-differential-mode oscillators. Future potential of differential-mode circuits is under great interests of many researchers. Therefore, we expect further development of these circuits especially with reconnection-less reconfigurations.

\section{REFERENCES}

[1] O. Casas, R. Pallas-Areny, "Basics of analog differential filters", IEEE Trans. Instrumentation and Measurement, vol. 45, no. 1, pp. 977-985, 1996. [Online]. Available: http://dx.doi.org/10.1109/ 19.481347

[2] A. A. El-Adawy, A. M. Soliman, H. O. Elwan, "A novel fully differential current conveyor and applications for analog VLSI", IEEE Trans. Circuits and Systems - II: Analog and Digital Signal Processing, vol. 47, no. 4, pp. 306-313, 2000. [Online]. Available: http://dx.doi.org/10.1109/82.839666

[3] S. M. Al-Shahrani, "Fully differential second order filter", in Proc. $4^{\text {th }}$ IEEE Int. Midwest Symposium on Circuits and Systems (MWSCAS), Hiroshima, Japan, 2004, pp. 299-301. [Online] Available: http://dx.doi.org/10.1109/MWSCAS.2004.1354353

[4] P. Beg, I. A. Khan, S. Maheshwari, M. A. Siddiqi, "Digitally programmable fully differential filter", Radioengineering, vol. 20, no. 4, pp. 917-925, 2011.

[5] R. Beg, S. Maheshwari, M. A. Siddigi, "Digitally controlled fully differential voltage- and transadmittance-mode biquadratic filter", IET Circuits, Devices and Systems, vol. 7, no. 4, pp. 193-203, 2013. [Online]. Available: http://dx.doi.org/10.1049/iet-cds.2012.0244

[6] M. A. Ibrahim, H. Kuntman, "A novel high CMRR high input impedance differential voltage-mode KHN-biquad employing DODDCCs", AEU - International Journal of Electronics and Communications, vol. 58, no. 6, pp. 429-433, 2004. [Online]. 
Available: http://dx.doi.org/10.1078/1434-8411-54100266

[7] A. K. Singh, P. Kumar, "A novel fully differential current mode universal filter", in Proc. 57th Int. Midwest Symposium on Circuits and Systems (MWSCAS), Texas, USA, 2014, pp. 579-582. [Online] Available: http://dx.doi.org/10.1109/MWSCAS.2014.6908481

[8] J. Koton, N. Herencsar, O. Sladok, J-W. Horng, "Pseudo-differential second-order band-reject filter using current conveyors", $A E U$ International Journal of Electronics and Communications, vol. 70, no. 6, pp. 814-821, 2016. [Online]. Available: http://dx.doi.org/ 10.1016/j.aeue.2016.03.009

[9] J. Koton, N. Herencsar, J. W. Horng, "Differential second-order voltage-mode all-pass filter using current conveyors", Elektronika ir Elektrotechnika, vol. 22, no. 5, pp. 52-57, 2016. [Online]. Available: http://dx.doi.org/10.5755/j01.eie.22.5.16344

[10] M. A. Ibrahim, S. Minaei, H. Kuntman, "DVCC based differentialmode all-pass and notch filters with high CMRR", Int. Journal of Electronics, vol. 93, no. 4, pp. 231-240, 2006. [Online]. Available: http://dx.doi.org/10.1080/00207210600562181

[11] M. S. Ansari, G. S. Soni, "Digitally-programmable fully-differential current-mode first-order LP, HP and AP filter sections", in Proc. Int. Conf. Signal Propagation and Computer Technology (ICSPCT), Ajmer, India, 2014, pp. 524-528. [Online]. Available: http://dx.doi.org/10.1109/ICSPCT.2014.6884963

[12] J. W. Horng, C. M. Wu, N. Herencsar, "Fully differential first-order allpass filters using a DDCC", Indian J Eng Mater Sci, vol. 21, no. 4, pp. $345-350,2014$

[13] I. A. Khan, M. I. Masud, S. A. Moiz, "Reconfigurable fully differential first order all pass filter using digitally controlled CMOS DVCC", in Proc. 8th IEEE GCC Conf. and exhibition (GCCCE), Muscat, Oman, 2015, pp. 1-5. [Online]. Available: http://dx.doi.org/10.1109/IEEEGCC.2015.7060082

[14] N. Herencsar, J. Jerabek, J. Koton, K. Vrba, S. Minaei, I. C. Goknar, "Pole frequency and pass-band gain tunable novel fully-differential current-mode all-pass filter", in Proc. IEEE Int. Symposium on Circuits and Systems (ISCAS), Lisbon, Portugal, 2015, pp. 26682671. [Online]. Available: http://dx.doi.org/10.1109/ISCAS. 2015.7169235

[15] R. Sotner, J. Jerabek, N. Herencsar, T. Zak, W. Jaikla, K. Vrba, "Modified current differencing unit and its application for electronically reconfigurable simple first-order transfer function", Advances in Electrical and Computer Engineering, vol. 15, no. 1, pp. 3-10, 2015. [Online]. Available: http://dx.doi.org/10.4316 /AECE.2015.01001

[16] R. Sotner, J. Jerabek, L. Langhammer, J. Polak, N. Herencsar, R. Prokop, J. Petrzela, W. Jaikla, "Comparison of two solutions of quadrature oscillators with linear control of frequency of oscillation employing modern commercially available devices", Circuits, Systems and Signal Processing, vol. 34, no. 11, pp. 3449-3469, 2015. [Online]. Available: http://dx.doi.org/10.1007/s00034-015 0015-7 